Supporting information

\title{
Surface roughness and substrate stiffness synergize to drive cellular mechanoresponse
}

Yong Hou ${ }^{1}$, Leixiao Yu ${ }^{1 *}$, Wenyan Xie ${ }^{2}$, Luis Cuellar Camacho ${ }^{1}$, Man Zhang ${ }^{3}$, Zhiqin

Chu ${ }^{4}$, Qiang $W^{3 *}{ }^{*}$, Rainer Haag ${ }^{1}$

${ }^{1}$ Institute of Chemistry and Biochemistry, Freie Universität Berlin, Takustr. 3, 14195

Berlin, Germany

${ }^{2}$ Institute of Pharmacy, Freie Universität Berlin, Königin-Luise-Str. 2+4, 14195

Berlin, Germany

${ }^{3}$ College of Polymer Science and Engineering, State Key Laboratory of Polymer

Materials and Engineering, Sichuan University, 610065 Chengdu, China

${ }^{4}$ Department of Electrical and Electronic Engineering, Joint Appointment with School of Biomedical Sciences, The University of Hong Kong, Pokfulam Road, Hong Kong, China

Email: leixiaoyu@zedat.fu-berlin.de,wei@scu.edu.cn 


\section{Materials}

All chemicals and solvents were reagent or HPLC grade, which were used as received and purchased from Sigma (Steinheim, Germany) unless stated otherwise. The deionized water used was purified using a Millipore water purification system with a minimum resistivity of $18.0 \mathrm{M} \Omega \cdot \mathrm{cm}$. The hyperbranched glycerol $(\mathrm{hPG})$ with $\mathrm{Mn}=$ $12000 \mathrm{~g} \mathrm{~mol}^{-1}$ and $\mathrm{Mw}=16000 \mathrm{~g} \mathrm{~mol}^{-1}$ was synthesized by a one-step ring-opening anionic polymerization (ROAP) as described in earlier publications. ${ }^{1}$ Amine-functionalized $\mathrm{hPG}\left(\mathrm{hPG}-\mathrm{NH}_{2}\right)$ was prepared according to previously published procedures of our group. ${ }^{2-3}$

\section{Synthesis of catecholic polyglycerol}
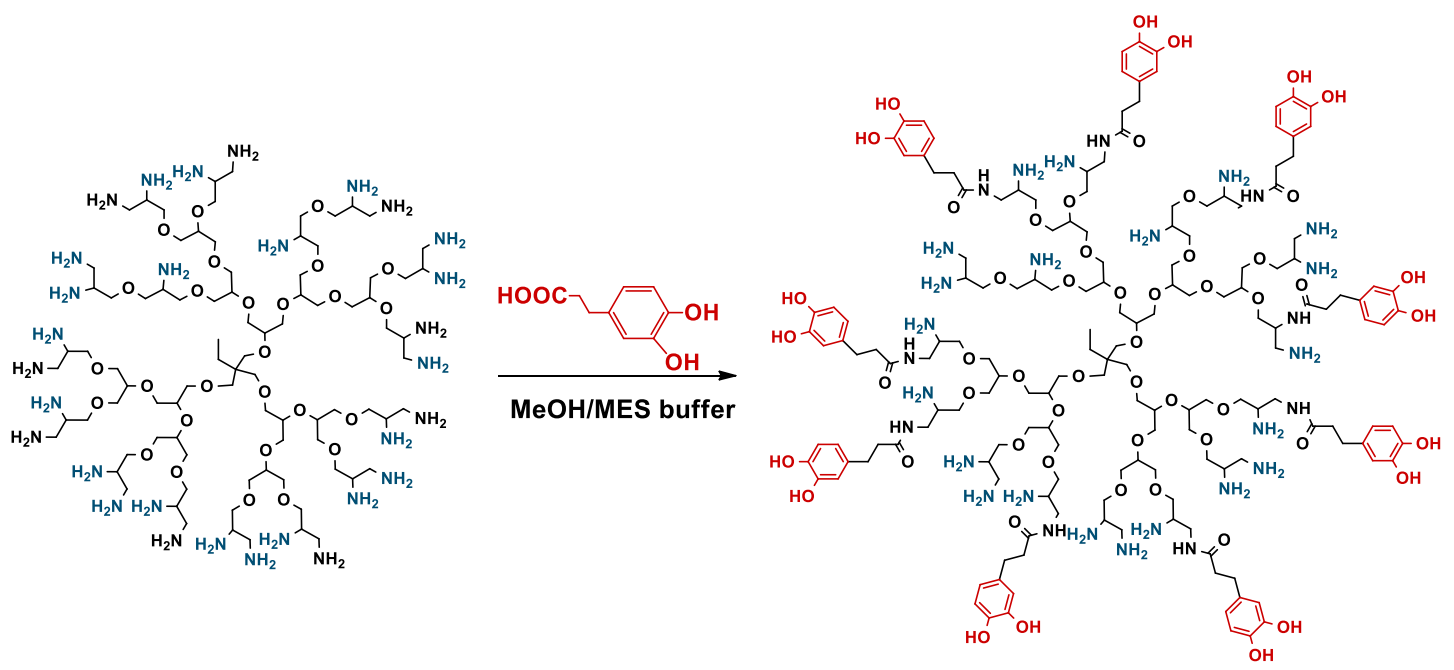

Figure S1. Scheme of the synthesis of catecholic polyglycerol.

As illustrated in Figure S1, $1 \mathrm{~g}$ of $\mathrm{hPG}-\mathrm{NH}_{2}\left(13.7 \mathrm{mmol}-\mathrm{NH}_{2}\right)$ and $3.93 \mathrm{~g}$ of 1-ethyl-3-(3-dimethylaminopropyl) carbodiimide hydrochloride (EDCI, 1.5 eqv. to amino groups) were dissolved in a mixture of $\mathrm{MeOH}$ and $\mathrm{pH} 4.8$ 2-(N-morpholino) ethanesulfonic acid (MES, $0.1 \mathrm{M})$ buffer $(60 \mathrm{~mL}, 1 \mathrm{v} / 1 \mathrm{v})$. Then $3.74 \mathrm{~g}$ of 3,4-dihdroxyhydrocinnamic acid (1.5 eqv. to amino groups) were added into the mixture and gently stirred overnight at room temperature. After removal of the solvent in a vacuum, the final residue was dialyzed in $\mathrm{MeOH}$ for 3 days. For higher stability and better storage, a small amount of $37 \% \mathrm{HCl}$ was added before drying the 
catecholic polyglycerol. ${ }^{1} \mathrm{H}-\mathrm{NMR}\left(500 \mathrm{MHz} ; \mathrm{CD}_{3} \mathrm{OD}\right): \delta=6.68-6.43(\mathrm{~m}, \mathrm{Ar})$; 4.21-3.02 (m, PG-backbone); 2.75 (m, $\left.\mathrm{COCH}_{2} \mathrm{CH}_{2} \mathrm{C}\right) ; 2.52$ (m, $\left.\mathrm{COCH}_{2} \mathrm{CH}_{2} \mathrm{C}\right)$ ppm. The grafting density of catechol groups to amino groups was calculated as $40 \%$ based on the ${ }^{1} \mathrm{H}-\mathrm{NMR}$ result.

\section{Synthesis of methacrylated gelatin (GelMA)}
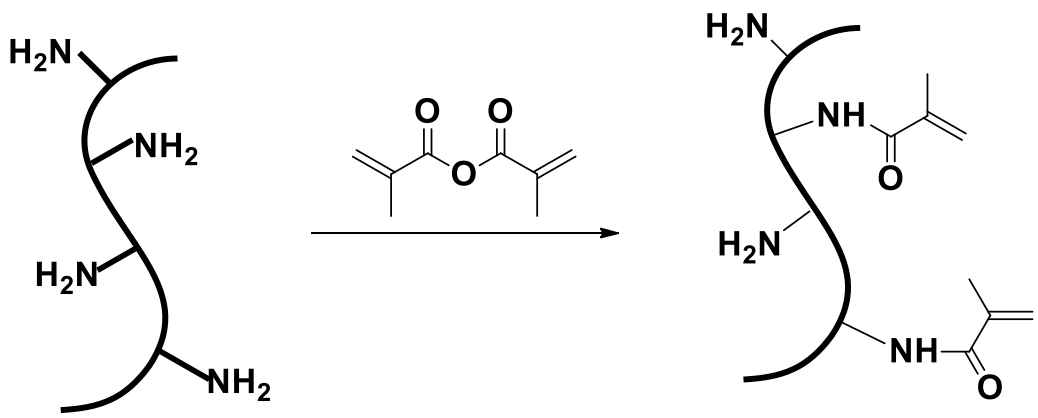

Figure S2. Scheme of the synthesis of methacrylated gelatin.

Methacrylated gelatin was synthesized as described procedure with a modification

(Figure S2). ${ }^{4}$ Briefly, $5 \mathrm{~g}$ type A porcine skin gelatin was dissolved into $50 \mathrm{~mL}$ Dulbecco's phosphate buffered saline (DPBS) at $60{ }^{\circ} \mathrm{C} .1 \mathrm{~mL}$ of methacrylic anhydride was then added into the gelatin solution dropwise under stirring at $50{ }^{\circ} \mathrm{C}$ and allowed to react for $2 \mathrm{~h}$. After quench the reaction by adding $100 \mathrm{~mL}$ pre-warmed $\left(40{ }^{\circ} \mathrm{C}\right) \mathrm{DPBS}$, the mixture was dialyzed against distilled water using $12-14 \mathrm{kDa}$ cutoff dialysis tubing for 4 days at $40{ }^{\circ} \mathrm{C}$. Finally, the water was removed via lyophilization to generate a white porous foam and stored at $-20{ }^{\circ} \mathrm{C}$ at argon atmosphere until further use. The grafting density of methacrylate was calculated from ${ }^{1} \mathrm{H}-\mathrm{NMR}$ results (Figure $\mathbf{S 3}, \mathrm{D}_{2} \mathrm{O}, 400 \mathrm{MHz}$ ) based a published method ${ }^{5}$ and it was $83.5 \%$ (to $\mathrm{NH}_{2}$ groups in gelatin chain). 


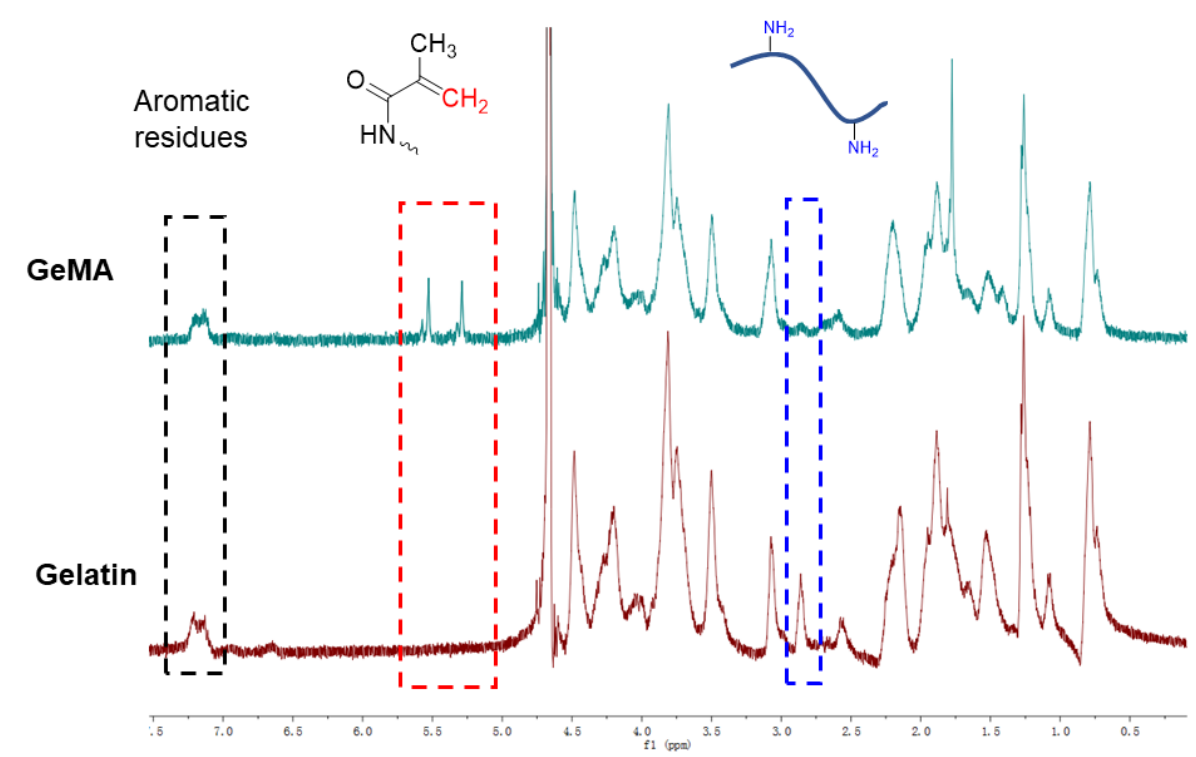

Figure S3. ${ }^{1} \mathrm{H}-\mathrm{NMR}$ spectra of unmodified gelation and methacrylated gelatin.

\section{Preparation of catecholic polyglycerol coating with roughness gradient}

The roughness gradient coatings were prepared via a one-step tilted dip-coating method. Generally, freshly cleaned glass slides into a solution of $0.5 \mathrm{mg} / \mathrm{mL}$ catecholic polyglycerol in a mixture of $\mathrm{MeOH}$ and 3-(N-morpholino)-propanesulfonic acid (MOPS, 0.1M, pH 8.6) buffer (v/v 4:1) at room temperature overnight. The depth of the solution was $2 \mathrm{~cm}$. The slides were tilted in the coating solution as shown in Scheme 1. After that, the coated slides were thoroughly rinsed with methanol and Milli-Q water, dried by $\mathrm{N}_{2}$ stream, and fully dried in vacuum.

\section{Surface morphology transfer printing onto polydimethylsiloxane (PDMS) stamp}

The PDMS stamp was prepared via the soft lithography. Firstly, the silicone elastomer (Dow Sylgard 184) and the hardener (Dow Sylgard 184 silicone elastomer curing agent) were mixed in a plastic beaker in the weight ratio: elastomer/curing agent=10:1. The elastomer and curing agent mixture together with the plastic beaker were then transfer into a "Thinly Mixer" machine (THINKY ARE-250 Mixing and Degassing Machine, Conditioning Planetary Mixer, program: $2 \mathrm{~min}, 1500 \mathrm{rmp}$ for mixing; $1 \mathrm{~min}$, 1500rmp for degassing, respectively). Then, the PDMS prepolymer was poured into a petri dish containing the catecholic PG coated surface roughness gradient slides. Put the petri dish inside a desiccator and turned on the pump to remove the air bubbles thoroughly, especially the ones directly located above the coating surface. Lastly, the petri dish was put into a preheated oven and kept the prepolymer curing at $65{ }^{\circ} \mathrm{C}$ for 
2h. Took out the petri dish, cool down to room temperature and gently peeled out the cured PDMS from catecholic PG coated slides to obtain the printed PDMS stamp. The printed PDMS stamp was immersed into DMF solution and sonication for $20 \mathrm{~min}$ to totally remove the catecholic $\mathrm{PG}$ residue and dried with $\mathrm{N}_{2}$ stream.

\section{Preparation of GelMA hydrogel with surface roughness gradient}

Interreference lithography engineering was used to fabricate the surface roughness gradient GelMA hydrogel. Briefly, the freeze-dried GelMA macromer was dissolved into DPBS containing $0.5 \%(\mathrm{w} / \mathrm{v})$ lithium phenyl-2,4,6-trimethylbenzoylphosphinate (LAP, photoinitiator) at $60{ }^{\circ} \mathrm{C} .500 \mu \mathrm{L}$ of the fully dissolved polymer solution was pipetted into the PDMS printed stamp mold, covered with a $1 \times 1 \mathrm{~cm}^{2}$ 3-(trimethoxysilyl) propyl methacrylate pre-treated glass coverslip, and exposed to 42 $\mathrm{mW} / \mathrm{cm}^{2} 365 \mathrm{~nm} \mathrm{UV} \mathrm{light} \mathrm{for} 2 \mathrm{~min}$. The samples were detached from the PDMS mold and incubated into DPBS for $24 \mathrm{~h}$ at $40{ }^{\circ} \mathrm{C}$ to remove the unreacted macromer and photoinitiator residues. The stiffness of the resulting GelMA hydrogel was adjusted by the initial concentration of GelMA macromers. For stiff GelMA hydrogel, the macromers concentration was set to $30 \%(\mathrm{w} / \mathrm{v})$ and the macromer concentration for soft GelMA hydrogel was 5\%.

\section{Atomic force microscopy (AFM)}

The AFM results were recorded by a MultiMode Nanoscope V scanning probe microscopy (SPM) system (Bruker, USA) in the air under ambient conditions. The commercially available AFM cantilever tips with a force constant of $\sim 48 \mathrm{~N} / \mathrm{m}$ and resonance vibration frequency of $\sim 330 \mathrm{kHz}$ were used, and the scanning rate was set at $0.8 \mathrm{~Hz}$. The AFM mode, Peak Force QNM, was used for better controlling the force with which the tip interacts with the surface. PPP-NCLR-20 probes with a force constant 21-98 N/m (silicon, resistivity: 0.01-0.02 $\Omega \mathrm{cm}$ Bruker) were used for the ambient measurement. The average surface roughness of the tested surfaces was calculated from 5 AFM images $\left(2 \times 2 \mu \mathrm{m}^{2}\right)$.

\section{Scanning electron microscope (SEM)}

The surface morphology of the catecholic PG gradient coatings and the printed PDMS stamps were investigated by a field emission scanning electron microscope (FE-SEM, Hitachi SU8030, Japan) at an accelerating voltage (Vacc) of $20 \mathrm{kV}$, a current of $10 \mu \mathrm{A}$. 
Before the measurement, all samples were thoroughly dried under high vacuum and sputtered a thin gold layer (8-10nm, Emscope SC 500, Quorum Technologies, UK).

\section{Rheology}

The stiffness of the GelMA hydrogel was measured by a universal stress Malvern rheometer kinexus lab (Germany) with a $20 \mathrm{~mm}$ cone-plate geometry. The testing gap was set according to previous reported protocol: load the fully swollen GelMA hydrogel, and lower the geometry until a plateau in the storage modulus ( $5 \mathrm{rad} / \mathrm{s}, 1 \%$ strain) was reached. ${ }^{6}$ To minimize the evaporation, the edge of the hydrogel was surrounded with a small amount of paraffin oil at the edges. The strain sweep experiments were conducted firstly at $5 \mathrm{rad} / \mathrm{s}$ with $0.01 \%-10 \%$ strain amplitude to determine the linear viscoelastic region of the tested GelMA hydrogel. Then the frequency sweep from 100-0.01 rad/s was performed at $1 \%$ strain amplitude. All the experiments were conducted at $37^{\circ} \mathrm{C}$.

\section{Traction Force Microscopy}

The hydrogels containing $0.5 \mu \mathrm{m}$ fluorescent carboxylated polystyrene beads (latex beads, carboxylate-modified polystyrene, fluorescent red, Sigma) for traction force microscopy were fabricated through a similar protocol as mentioned above with a small modification. Before the photo-crosslinking, $6.5 \mu \mathrm{L}$ dye labeled beads (L3280, Sigma) were added into $1 \mathrm{~mL}$ GelMA macromer/ LAP mixture. $500 \mu \mathrm{L}$ of the fully dissolved polymer solution was pipetted into the PDMS printed stamp mold, covered with a $1 \times 1 \mathrm{~cm}^{2} 3$-(Trimethoxysilyl) propyl methacrylate pre-treated glass coverslip, and exposed to $42 \mathrm{~mW} / \mathrm{cm}^{2} 365 \mathrm{~nm}$ UV light for $2 \mathrm{~min}$. The samples were detached from the PDMS mold and incubated into DPBS for $24 \mathrm{~h}$ at $40{ }^{\circ} \mathrm{C}$ to remove the unreacted macromer and photoinitiator residues. The hydrogel was loaded with cells and incubated for $24 \mathrm{~h}$. Fluorescence microscopy was used to take images of focused beads as well as images of focused spread cells. Then, the cells were treated using 1\% SDS (w/v) for over $15 \mathrm{~min}$ and washed away on the microscopy stage. The images were utilized for defining the original position of the dye-labeled beads. The obtained images were first aligned to correct for the experimental drift of the samples using the ImageJ plugin "Align slices in stack." The displacement field in a spread cell region was subsequently calculated by a "particle image velocimetry" plugin in ImageJ. The obtained result was reconstructed using the "plot particle image velocimetry" plugin 
in order to generate the displacement field as a vector plot.

\section{Surface roughness of the swollen GelMA hydrogel}

The stiffness of the swollen GelMA hydrogel is lower. Because of the soft GelMA hydrogel, it is especially very hard to obtain directly the surface roughness information from AFM testing. Therefore, the surface roughness of the fully swollen roughness gradient GelMA hydrogel was analyzed and calculated based on the 3D surface images constructed by confocal microscopy scan. Before testing, the GelMA hydrogels were incubated into a $10 \mu \mathrm{g} / \mathrm{mL}$ rhodamine B isothiocyanate DPBS solution at room temperature for $20 \mathrm{~min}$ to fluorescently label the hydrogel surface (the -NSC groups from $\mathrm{RhB}$ tend to react with $-\mathrm{NH}_{2}$ or $-\mathrm{OH}$ groups from the gelatin backbone). The dye-labeled hydrogel slides were then thoroughly rinsed with DPBS and the 3D surface imaging reconstruction was performed using a Leica SP8 microscope. A $63 \times$ oil immersion objective was used. Each sample was imaged using multiple z-stack images with each step of $0.1 \mu \mathrm{m}$. The amplitude heights of the profile 3D reconstruction hydrogel surface were measured and analyzed by Image J. The root-mean squired surface roughness $\left(\mathrm{R}_{\mathrm{q}}\right)$ and arithmetic average surface roughness $\left(R_{a}\right)$ were then calculated from the following equation:

$\mathrm{R}_{\mathrm{q}}$ :

$$
R_{q}=\sqrt{\frac{1}{n} \sum_{i=1}^{n} y_{i}^{2}}
$$

$\mathrm{R}_{\mathrm{a}}$ :

$$
R_{a}=\frac{1}{n} \sum_{i=1}^{n}\left|y_{i}\right|
$$

Where the $y_{i}$ is the vertical distance from the mean line to the $i^{\text {th }}$ data point.

\section{Cell culture}

MSCs were purchased from EMD Millipore (Darmstadt, Germany) and cultured in DMEM (Gibco 11965092) supplemented with 10\% bovine growth serum (Gibco 16030074) and $1 \%$ penicillin/streptomycin (Gibco 15140122 ) at $37{ }^{\circ} \mathrm{C}$ with $5 \% \mathrm{CO}_{2}$. The osteogenic differentiation medium (C-28013) was obtained from Promo Cell (Heidelberg, Germany). The cells were passaged twice a week according to the 
standard protocols. The fourth to sixth passages of MSCs were used in this study.

\section{Immunocytochemistry}

After culturing on surface roughness gradient GelMA hydrogel surface in DMEM medium for set time, the cells were fixed with $4 \%$ formaldehyde for $30 \mathrm{~min}$ at room temperature, permeabilized with $0.25 \%$ Triton $\mathrm{X}-100$ in PBS for $10 \mathrm{~min}$. The non-specific binding epitopes were blocked with 1\% BSA in PBST (PBS with $0.1 \%$ Triton X-100) for $45 \mathrm{~min}$ at room temperature. Next, the cells were incubated with primary antibodies overnight at $4{ }^{\circ} \mathrm{C}$. The primary antibodies used in this work are: mouse monoclonal anti-Paxillin (BD Transduction Laboratories ${ }^{\mathrm{TM}}$ 612405, 1:100 dilution), rabbit monoclonal anti-YAP (cell signaling 4912S, 1:200 dilution), rabbit monoclonal anti-Phospho-FAK (Tyr397) (Thermo 700255, 1:300 dilution), mouse monoclonal anti-lamin A/C (Thermo MA3-1000, 1:100 dilution). After the incubation, cells were washed with PBS two times and then incubated with the appropriate secondary antibodies (Invitrogen, goat anti-mouse IgG Alexa Fluor 488 A-11029 or goat anti-rabbit Alexa Fluor 568 A-11011, 1:500 dilution) for $1 \mathrm{~h}$ at room temperature. Finally, the cells were stained with DAPI (Sigma D9542, 1:1000 dilution) and Phalloidin-iFluor 647 (Abcam ab176759, 1:1000 dilution) and imaged by Zeiss Axio Observer Z1 phalloidin microscope (Zeiss, Germany) or a confocal microscope (SP8, Leica).

\section{MSC differentiation and alkaline phosphatase (ALP) staining and quantification}

The MSCs were seeded on roughness gradient soft and stiff GelMA hydrogel surfaces in basal growth medium with density of 5000 cell $/ \mathrm{cm}^{2}$ for $24 \mathrm{~h}$. Afterwards the medium was replaced by standard osteogenic differentiation medium for one week. The culture medium was replaced every 3 days. The cells were then fixed in $4 \%$ paraformaldehyde for $5 \mathrm{~min}$ and stained by Fast Blue RR/naphthol (Sigma) to quantify osteogenic differentiation. Afterwards, DAPI was added to mark the nuclei. The stained cells were rinsed in Milli-Q water and observed in the inverted microscope mounted with a color camera (Zeiss Axio Observer Z1, Germany). Micrographs were captured for post statistical analysis. The fraction of osteogenesis of cells on different roughness regions were calculated by counting the number of cells in more than three randomly selected fields (10× magnification) and normalizing to the total number of cells detected by DAPI staining in each individual sample. 


\section{Statistical analysis}

Data are represented as mean \pm standard deviation (S.D). Group differences were conducted by one-way ANOVA with Tukey's post -hoc test. P-values $<0.05$ were considered as statistically significant $\left({ }^{*} \mathrm{p}<0.05,{ }^{* *} \mathrm{p}<0.01, * * * \mathrm{p}<0.001,{ }^{* * * *} \mathrm{p}<\right.$ 0.0001). All statistical analyses were performed with GraphPad Prism 8. 


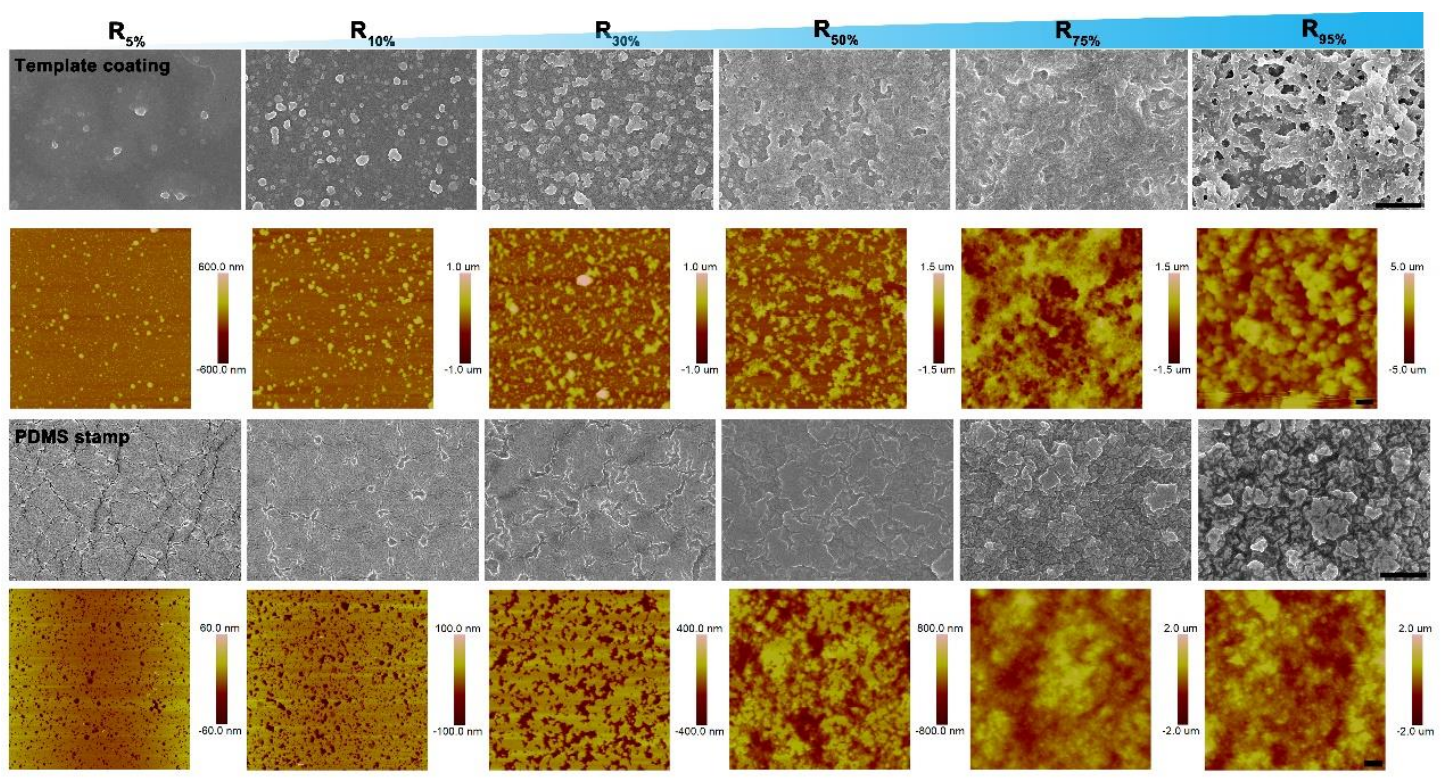

Figure S4. Surface morphology of the roughness gradient catecholic PG coating (top) and the transfer - printed PDMS stamp prepared via soft lithography (down). The scale bar for SEM images and AFM images indicate $5 \mu \mathrm{m}$. 

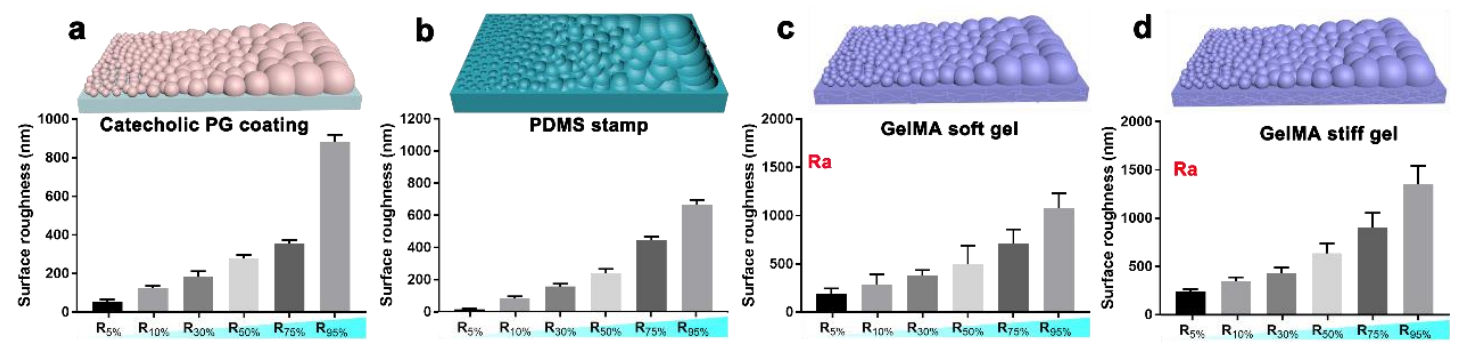

Figure S5. The surface roughness $\left(\mathrm{R}_{\mathrm{q}}\right)$ of the template roughness gradient catecholic PG coating (a) and (b) the corresponding transfer printed PDMS stamp. These $\mathrm{R}_{\mathrm{q}}$ values were obtained and quantified from AFM measurements. (c) The calculated average surface roughness $\left(R_{a}\right)$ of fully swollen soft and (d) stiff GelMA roughness gradient hydrogel. These $\mathrm{R}_{\mathrm{a}}$ data were analyzed and calculated based on the $3 \mathrm{D}$ surface images constructed by confocal microscopy scan as aforementioned methods. 


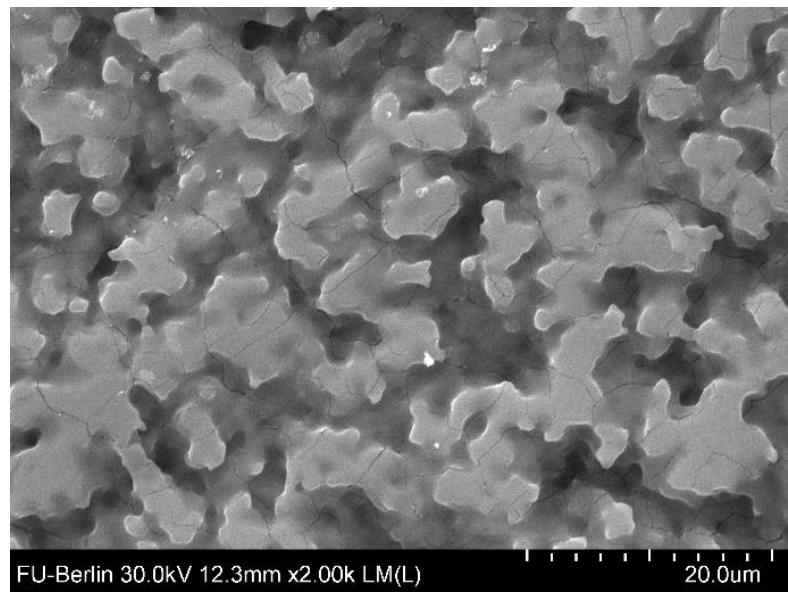

Figure S6. Surface morphology of the transfer - printed PDMS stamp (representative image at $\mathrm{R}_{95 \%}$ position) after hydrogel detachment. There was no GelMA left on the PDMS mold and the structure remained stable after being reused for several times $(>3)$. 


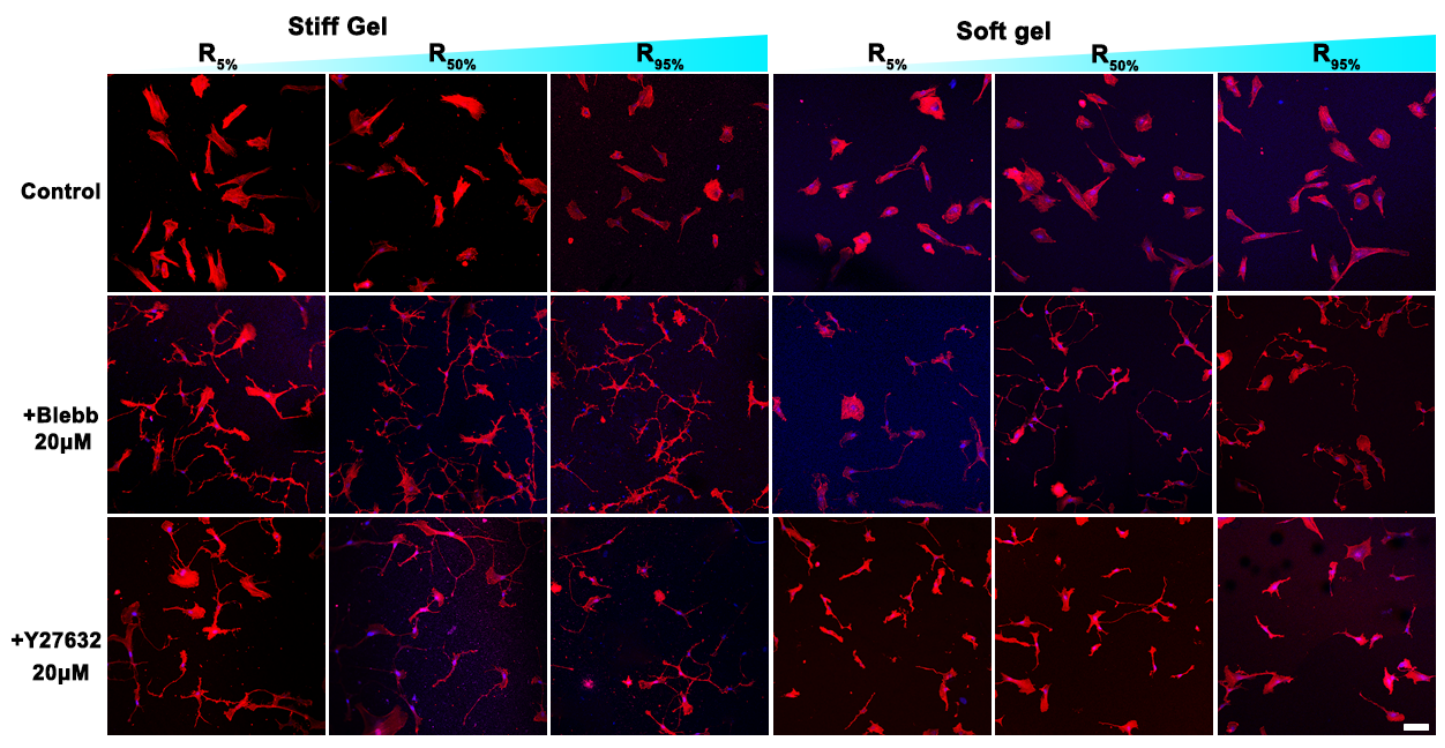

Figure S7. Representative images of F-actin staining and spread area of MSCs from $\mathrm{R}_{5 \%}, \mathrm{R}_{50 \%}$, and $\mathrm{R}_{95 \%}$ positions on stiff (right,) and soft gel (left) treated with blebblstain (middle) and Y27632 (down) for 1 day. The inhibitor concentration is 20 $\mu \mathrm{M}$. The scale bar indicates $100 \mu \mathrm{m}$.

Reference:

1. Burakowska, E.; Haag, R., Dendritic polyglycerol core-double-shell architectures: synthesis and transport properties. Macromolecules 2009, 42, 5545-5550.

2. Roller, S.; Zhou, H.; Haag, R., High-loading polyglycerol supported reagents for Mitsunobu-and acylation-reactions and other useful polyglycerol derivatives. Mol. Diversity 2005, 9, 305-316.

3. Wei, Q.; Achazi, K.; Liebe, H.; Schulz, A.; Noeske, P. L. M.; Grunwald, I.; Haag, R., Mussel inspired dendritic polymers as universal multifunctional coatings. Angew. Chem., Int. Ed. 2014, 53 , 11650-11655.

4. Van Den Bulcke, A. I.; Bogdanov, B.; De Rooze, N.; Schacht, E. H.; Cornelissen, M.; Berghmans, H., Structural and rheological properties of methacrylamide modified gelatin hydrogels. Biomacromolecules 2000, 1, 31-38.

5. Hoch, E.; Hirth, T.; Tovar, G. E.; Borchers, K., Chemical tailoring of gelatin to adjust its chemical and physical properties for functional bioprinting. J. Mater. Chem. B 2013, 1 (41), 5675-5685.

6. Meyvis, T.; De Smedt, S.; Demeester, J.; Hennink, W., Rheological monitoring of long-term degrading polymer hydrogels. J. Rheol. 1999, 43, 933-950. 